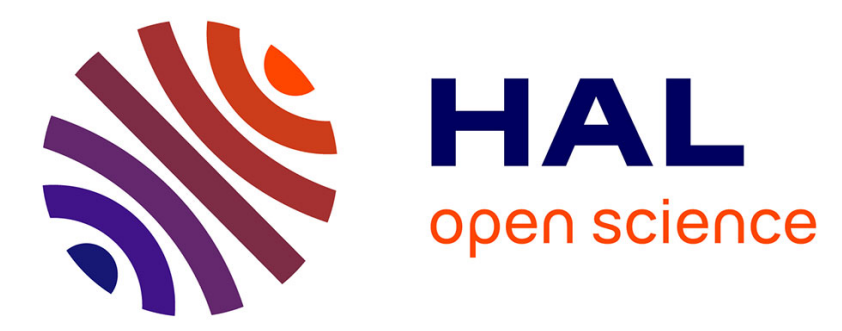

\title{
To portray clonal evolution in blood cancer, count your stem cells
}

\author{
Anne-Marie Lyne, Lucie Laplane, Leïla Perié
}

\section{To cite this version:}

Anne-Marie Lyne, Lucie Laplane, Leïla Perié. To portray clonal evolution in blood cancer, count your stem cells. Blood, 2020, 10.1182/blood.2020008407 . hal-03097640

HAL Id: hal-03097640

https://cnrs.hal.science/hal-03097640

Submitted on 25 Mar 2021

HAL is a multi-disciplinary open access archive for the deposit and dissemination of scientific research documents, whether they are published or not. The documents may come from teaching and research institutions in France or abroad, or from public or private research centers.
L'archive ouverte pluridisciplinaire HAL, est destinée au dépôt et à la diffusion de documents scientifiques de niveau recherche, publiés ou non, émanant des établissements d'enseignement et de recherche français ou étrangers, des laboratoires publics ou privés. 


\section{To portray clonal evolution in blood cancer, count your stem cells}

Running title: Clonal evolution in hematological malignancies

Anne-Marie Lyne ${ }^{1,2}$, Lucie Laplane ${ }^{3,4 *}$, Leïla Perié ${ }^{1,2 *}$

1. Institut Curie, PSL Research University, CNRS UMR168, Paris, France.

2. Sorbonne Universités, UPMC Univ Paris 06, France

3. Université Paris I Panthéon-Sorbonne, CNRS UMR8590 IHPST, Paris, France

4. Gustave Roussy, UMR1287, Villejuif, France

* Co-last authors

Corresponding authors:

Lucie Laplane

IHPST,

13 rue du Four

75006 Paris

Phone: +33143546036

Fax: +33143252948

email: lucie.laplane@univ-paris1.fr

Leïla Perié

Institut Curie, CNRS UMR168

11 rue Pierre et Marie Curie

75005 Paris

Phone: +33 156246229

email: leila.perie@curie.fr

Metrics:

Text: 3724/4000

Number of figures: 3

Number of references: 97/100 


\section{Abstract:}

Clonal evolution, the process of expansion and diversification of mutated cells, plays an important role in cancer development, resistance and relapse. While clonal evolution is most often conceived of as driven by natural selection, recent studies uncovered that neutral evolution shapes clonal evolution in a significant proportion of solid cancers. In hematological malignancies, the interplay between neutral evolution and natural selection is also disputed. Because natural selection selects cells with a higher fitness - providing a growth advantage to some cells relative to others - the architecture of clonal evolution serves as indirect evidence to distinguish natural selection from neutral evolution and has been associated with different prognoses for the patient. Linear architecture, when the new mutant clone grows within the previous one, is distinctive of hematological malignancies and typically interpreted as driven by natural selection.

Here we wish to discuss the role of natural selection and neutral evolution in the production of linear clonal architectures in hematological malignancies. While it is tempting to attribute linear evolution to natural selection, we argue that a lower number of contributing stem cells accompanied by genetic drift can also result in a linear pattern of evolution as illustrated by simulations of clonal evolution in hematopoietic stem cells. The number of stem cells contributing to long-term clonal evolution is not known in the pathological context and we advocate that estimating these numbers in the context of cancer and ageing is crucial to parsing out neutral evolution from natural selection, two processes that require different therapeutic strategies. 


\section{Introduction}

During cancer development, mutations accumulate over time leading to the expansion and diversification of clones, cancer cells that share a specific set of mutations inherited from a common ancestor(box 1). This process, known as clonal evolution, plays an important role in cancer development, progression, therapy resistance and relapse. ${ }^{1,2}$

Clonal evolution is increasingly depicted as an evolutionary process driven by natural selection (box 1 ). In this framework, clones expand and out-compete other clones due to the fitness advantage conferred by their mutations. The mutations that increase fitness and confer a clonal growth advantage are called driver mutations. They represent a crucial target for cancer treatment and are subject to many clinical and biological investigations. ${ }^{3-}$ 5 Of note, a driver mutation can be selected through tumorigenesis but need not be required for cancer maintenance. In parallel, cells also accumulate neutral mutations, called passenger mutations, which confer no fitness advantage and are therefore not subject to natural selection. Neutral evolution of these passenger mutations can also shape clonal evolution, notably by a phenomenon called genetic drift in which the allele frequencies of a mutation change over time due to mere chance (box 1). Additionally, when passenger mutations occur in the same cells as driver mutations, the passenger mutations increase their allele frequency with the driver mutations. This phenomenon, called hitchhiking (box 1), can also participate in clonal evolution. Exactly how the interplay of neutral evolution and natural selection shapes clonal evolution in cancer is disputed. Indeed, although natural selection is the dominant view, different studies have uncovered that neutral evolution shapes clonal evolution in more cases of cancer than expected. ${ }^{6-8}$ Although it seems counter-intuitive that malignant hematological phenotypes such as aberrant growth could be associated with neutral evolution, it is possible that mutations that are neutral in the stem cells gain a selective advantage in other hematopoietic compartments, resulting in cancerous aberrant growth. For example, in some chronic myelomonocytic leukemia (CMML) patients, certain mutated clones expand more in myeloid differentiating cells than in stem cells due to increased sensitivity to GM-CSF. 9,10 In hematological malignancies, the role of neutral evolution has been less investigated except in the case of multiple myeloma, where it has been linked to a poor prognosis. ${ }^{11}$

Distinguishing driver from passenger mutations is not always straightforward. Functional assays to test the impact of mutations on cell fitness are the gold standard but they are cumbersome to carry out on each mutation. Alternatively, recurrent mutations across patients with the same cancer type is a sign of natural selection but difficulties in mutation calling ${ }^{12-14}$ and neutral mutational hotspots complicate this inference. ${ }^{15,16}$ In other words, some recurrent mutations can look like driver mutations because they are occurring more frequently. Lastly, the architecture of clonal evolution can serve as indirect evidence of natural selection or neutral evolution. Clonal architecture is described as linear when the new mutant clone grows within the previous one (Figure $1 \mathrm{~A})$ and branched when multiple mutant clones arise independently and grow in parallel (Figure 1B). Of note, classification as linear or branched architecture might vary if the mutations are detected by using targeted sequencing or whole exome sequencing, as more mutations are recovered with whole exome sequencing resulting in more complex architecture. A linear architecture is typically interpreted as the output of natural selection while neutral evolution has been described as an extreme case of branched evolution.6,7,17-19 Intriguingly, while branched evolution has 
been reported in both solid tumors and hematological malignancies, most if not all cases of linear evolution reported so far are in hematological malignancies. ${ }^{10,20-28}$

Importantly, clonal architecture has been associated with different prognoses and impacts clinical outcome. In chronic lymphocytic leukemia, an increased number of sub-clonal driver mutations was associated with an inferior failure-free survival while an increase of clonal drivers was not. ${ }^{29}$ In acute myeloid leukemia, clonal dominance has been associated with a worse prognosis, ${ }^{30}$ while branched evolution of signaling mutations conveyed an inferior event-free survival compared to linear evolution. ${ }^{31}$ These various associations between clonal architecture and clinical outcomes highlight the importance of understanding the underlying processes that drive clonal architecture. While most reports of linear evolution implicitly assume natural selection, the role of neutral evolution in shaping linear clonal architectures has not been investigated.

Here we wish to discuss the role of natural selection and neutral evolution in the production of linear clonal architectures in hematological malignancies. While it is tempting to attribute linear evolution to natural selection, we argue that a lower number of contributing stem cells (SCS) accompanied by drift can also result in a linear pattern of evolution as illustrated by simulations of clonal evolution in hematopoietic stem cells (HSCs). The number of SCs contributing to long-term clonal evolution is not known in the pathological context and we advocate that estimating these numbers in the context of cancer and ageing is crucial to parsing out neutral evolution from natural selection, two processes that require different therapeutic strategies.

\section{Box 1: Definitions}

Clone: Cells that share a specific set of mutations.

Linear evolution: A Russian doll organization of clones where each new mutant clone grows within the previous one.

Branched evolution: Multiple mutant clones arise independently and grow in parallel.

Natural selection: Process which changes allele frequencies across generations due to better adapted clones contributing more offspring to the next generation than less well adapted clones.

Neutral evolution: Evolution without natural selection i.e., the mutations introduced do not confer a functional advantage or disadvantage.

Genetic drift: Stochastic process in which allele frequencies change across generations due purely to chance, e.g., because one clone randomly has more cell death than another. It occurs in all finite populations, but has largest effect in small populations.

Hitchhiking: Process which allows neutral or weakly deleterious mutations to reach high frequency due to their presence in a clone which also has one or more driver mutations.

Effective population size: The cells that effectively contribute to clonal evolution. 


\section{A role for neutral evolution in linear architecture of hematological malignancies?}

While linear evolution seems to be a hallmark of hematological malignancies, both linear and branched evolution have been reported for patients with the same hematological malignancies (e.g. ${ }^{10,20,23,24}$ ), and patients sometimes transition from one type to the other. ${ }^{32-34}$ Little is known about what differs between patients with linear and branched evolution, and understanding which of natural selection or neutral evolution mechanistically drive linear evolution has never been discussed. Given that linear evolution can result in more favorable outcomes for patients, ${ }^{29,31}$ this discussion is of importance for the design of therapeutic interventions. ${ }^{35}$

Linear evolution follows a pattern that intuitively looks consistent with a model of natural selection (Figure $1 \mathrm{~A}$ ): each new driver mutation provides a selective advantage enabling those cells to outcompete previous clones, resulting in sequential dominant clones that expand and sometime sweep the whole compartment. Figure 2 illustrates this scenario using a toy model to simulate clonal evolution in the HSC population (see method). When the fitness advantage conferred by each mutation increases (here their probability to self-renew), the linearity index and the final clone sizes increase (Figure 2A and 2B). When mutations confer a strong selective advantage, the result is linear evolution similar to that observed in patients (Figure 2C).

However, we argue that the hypothesis that linear evolution could also come from neutral evolution must not be discarded too rapidly. The neutral evolution model is usually presented as an extreme case of branched evolution (e.g. ${ }^{6,19}$ ) in which random mutations with no fitness advantage accumulate over time, driving clonal evolution in the absence of any natural selection. ${ }^{36}$ Nevertheless, this representation results from the hidden assumption that the population of cells that effectively contributes to clonal evolution, called the "effective population size" in population genetics, is large. Indeed, in large populations, in the absence of natural selection, clones rarely expand to a large frequency and it is therefore likely that new mutations occur outside of pre-existing clones. In contrast, it is well established that effective populations of small size are more susceptible to genetic drift, in which some clones expand more than others by mere chance (i.e. the expansion of clones is not due to an increased fitness). ${ }^{37,38}$ In hierarchical models such as blood where only SCs can self-renew and give rise to all the other cells, clonal evolution is driven by $\mathrm{SCs}^{39}$ a small fraction of the cancer cells (in evolutionary terms, the effective population size is incredibly small compared to the census population size). Therefore, it is possible that a low number of contributing SCs in blood cancer could favor linear clonal evolution due to clones being more subject to genetic drift, i.e. a particular case of neutral evolution in a roughly constant sized population where some clones grow bigger by mere chance. Figure 2D-F illustrates this point using the same toy model as before, this time removing the selective advantage conferred by mutations and instead varying the number of SCs. The linearity index increases with a smaller number of contributing SCs (Figure 2D). Simulations of small SC compartments frequently develop sizable clones (Figure $2 \mathrm{E}$ ) and in some cases present examples of linear evolution similar to the patterns observed in patients (Figure 2F) (e.g. $\left.{ }^{10,21,22,24-26}\right)$. When the number of stem cells increases, the impact of genetic drift becomes minor. In summary, while linear evolution seems to be driven by natural selection, it can also result from neutral evolution through genetic drift if the number of contributing SCs is sufficiently low.

In practice, others have reported a role for neutral evolution in 17 to $20 \%$ of patients with multiple myeloma, and this was associated with a shorter survival. ${ }^{11}$ However, in this study neutral evolution was not linked to clonal 
architecture which is complex in this disease. ${ }^{40}$ Applying the same approach and R-squared threshold 8 to 17 whole genome sequenced CMML patient samples, ${ }^{23}$ a type of leukemia in which linear evolution is frequent, ${ }^{10,21}$ we found that 4 of the 17 patients fit the neutral evolution model (UPN57, 62, 64, 65; Figure 3A), similar to the proportion in multiple myeloma. ${ }^{11}$ In this CMML cohort, patient UPN62 had 6 exonic mutations, among which 5 potential drivers (Figure 3B) organized in a linear clonal architecture (Figure 3C), making it a good candidate for clonal evolution driven by natural selection. Yet, the R-squared of 0.9967 also indicates that the mutations subsequent to the TET2 mutation occurred through neutral evolution. This example illustrates that neutral evolution is a plausible interpretation of clonal evolution in some patients with myeloid malignancies. Separating neutral evolution and natural selection is an oversimplification as both mechanisms occur in parallel. It is possible that neutral evolution and natural selection successively drive clonal evolution. ${ }^{41}$ For example, in linear evolution, the first mutation could be a driver and increase the fitness of the clone, and subsequent mutations could be neutral and confer no growth advantage over that of the first. Many different combinations of natural selection and neutral evolution can be envisaged.

\section{Is the number of contributing HSCs compatible with genetic drift?}

As neutral evolution can produce linear architecture when the population of long-term contributing SCs is small, estimating the number of contributing SCs is crucial to assess the potential role for neutral evolution in hematological malignancies. More precisely, for genetic drift to play a significant role in SCs within the timescale of a human lifetime, it is not the absolute number of SC that matters but the number of SC multiplied by the time between symmetric divisions. ${ }^{42,43}$ Of course, to contribute to cancer the SCs that have undergone genetic drift also need to differentiate into blasts or more mature cells.

In healthy individuals, current estimates of HSCs are derived from indirect evidence, for example, the detection of somatic mutations in downstream cells in one individual, ${ }^{42}$ and span from as few as 385 active HSCs in steady state, ${ }^{44}$ to as many as 50000 to 600000 HSCs in adult hematopoiesis. ${ }^{42,43,45,46}$ While the former estimates leave space for neutral linear evolution, the latter render it implausible.

In hematological malignancies, there are a number of unknowns that should make us cautious before ruling out neutral evolution. First, whilst there are a few estimates of HSC number for healthy individuals, they are not necessarily applicable to clonal evolution in cancer. In the context of hematological malignancies, it is unknown whether the number of CSCs contributing to clonal evolution is similar to the number of HSCs in healthy hematopoiesis and how this depends on the type of malignancy. In addition, cancer clones start from single mutated cells which multiply and expand over time, and therefore the early disease stage necessarily comprises a small number of cells. Genetic drift may therefore play a sizeable role during this time period. Secondly, both age and personal medical history may also considerably impact, either transiently or permanently, the size of the relevant HSC compartment. Several studies suggest decreased clonal diversity, 47,48 exhaustion of HSC functionality, $^{49-51}$ and increased functional heterogeneity among HSCs with age. ${ }^{48}$ The number of HSCS contributing to hematopoiesis in the elderly, the group most likely to develop myeloid malignancies, is unknown. In addition, the specific biological challenges encountered by an individual can also impact the number of HSCs. 
Amongst the challenges shown to reduce the number of HSCs or induce quiescence are obesity, ${ }^{52,53}$ viral and bacterial infection, ${ }^{54,55}$ autoimmune disease such as acquired aplastic anemia ${ }^{56}$ and total body irradiation, as well as radiotherapy and chemotherapy used in the treatment of solid cancers. ${ }^{57}$

All of these challenges that reduce HSC numbers have been associated with the occurrence of clonal hematopoiesis - the over-representation of a single clone in the blood or bone marrow. ${ }^{58-60}$ But the respective role of natural selection and neutral evolution in these clonal expansions has yet to be fully elucidated. The fact that synonymous (non-functional) mutations are rarely observed at high allele frequency in the blood of healthy people is good evidence in favor of natural selection in clonal hematopoiesis. ${ }^{43}$ However, whole genome sequencing has shown that clonal hematopoiesis in some patients occurs with no known driver mutations. ${ }^{61,62}$ This observation has led to different interpretations depending on the size of the stem cell population assumed or inferred. Assuming a relatively small number of stem cells and relying on low depth sequencing, Zink et al concluded that the allele frequencies of mutations were consistent with genetic drift. ${ }^{62}$ Conversely, Poon et al. concluded that there must be hidden driver mutations to explain the allele frequency distribution of synonymous mutations. ${ }^{63}$ In conclusion, current evidence points to a role for natural selection in clonal hematopoiesis but more evidence is needed before ruling out a role of neutral evolution.

Another interesting case is hematopoietic stem cell transplantation which results in a bottleneck in SC number. Only a limited number of cells initially seed and expand to regenerate the whole hematopoietic system, a situation which could be favorable to drift. Using the insertion site of the lentiviral vector used for gene therapy in HSCs from patients with Wiskott-Aldrich Syndrome, Biasco et al. estimated that 1,600-4,300 transduced HSCs were actively contributing to long-term hematopoiesis, ${ }^{64,65}$ a range which, particularly at the lower end, could allow a role for genetic drift. Interestingly, clonal hematopoiesis from donors can be engrafted in patients, ${ }^{66-69}$ the likelihood of a clone persistently engrafting is not dependent on the donor allele frequency, ${ }^{66}$ and there is no clear relationship between clone size in the recipient and that in the donor. ${ }^{66,68}$ These discrepancies between donor and recipient can be attributed to a change in the selection pressure due to transplantation or variability due to drift. In addition to uncertainties in the number of HSCs in various physiological contexts, it has been extensively shown that HSCs are heterogeneous in their functional properties, for example, in their differentiation potential, ${ }^{70-72}$ cell cycle time, ${ }^{73}$ downstream cellular output, ${ }^{73-76}$ and level of quiescence. ${ }^{70,77}$ The heterogeneity in HSC properties has several implications for the estimation of the SC effective population size. SCS (CSCs or HSCS) that exhaust their self-renewal potential due to a limit on the number of divisions, ${ }^{78}$ or SCs that stay quiescent throughout the period of interest cannot make relevant contributions to clonal evolution. ${ }^{79}$ If SCs have stable but heterogeneous output, the relevant SC pool may be different for different malignancies. ${ }^{72}$ More fundamentally, recent studies have led to intense debate over the contribution of HSCs in homeostatic hematopoiesis, with some studies suggesting that they are largely dispensable. ${ }^{80-84}$ Similar investigations remain to be performed in hematological malignancies. Lastly, although progress in live imaging has started to shed light on the spatial distribution and motility of stem cells in the bone marrow, 85 the impact of cell mixing dynamics on clonal evolution remains unexplored and is likely to impact our interpretation of genomic data. 
While the effective population size in HSCs and CSCs remains difficult to address and is largely unknown, it is clear that it can vary widely depending on context and life history. These variations might impact both the processes that drive clonal evolution and the resulting clonal architecture by making linear evolution more or less likely to occur through genetic drift. To determine whether and when clonal evolution in hematological malignancies is mainly driven by natural selection or neutral evolution, it is crucial to determine the number of SCs actively contributing to clonal evolution.

\section{Implications of potential genetic drift for cancer treatment}

Distinguishing whether natural selection and/or neutral evolution play a role in clonal evolution has some consequences for the design of therapeutic interventions. In particular, it might be important in the case of minimal residual disease and relapse to determine whether the residual clone is made of cells that are intrinsically resistant to the treatment or escape for some other reason. ${ }^{86}$ Some therapeutic strategies to handle these issues rely on the assumption of natural selection. For example, adaptive therapies aim at maintaining clonal competition in order to avoid therapy escape, 87,88 and evolutionary steering strategies aim at steering the tumor evolution with a first drug and then use a second drug to target Darwinian adaptation trade-off, i.e. specific sensitivity associated with resistance to the first drug. ${ }^{89}$ Both are elegant solutions to avoid the issue of therapy escape, but both also rely on exploiting selective pressures or competition between clones and thus would be ineffective in cases where intra-tumor diversity is dominated by neutral evolution. It is unclear whether neutral evolution can persist during treatment, whether treatment always introduces selective pressure which can be exploited to design treatment, or whether it creates a bottleneck even more favorable to genetic drift. In the context of natural selection, understanding the selective pressures and associated fitness advantage of cancer cells can help us to predict future clonal evolution and choose the appropriate treatment. In contrast, clones undergoing neutral evolution in small populations are subject to stochastic amplification or reduction in a less predictable way which complicates the design of therapeutic intervention. It is possible that patients with the same hematological malignancies undergo different evolutionary processes, some mainly driven by natural selection and others undergoing neutral evolution. These different underlying processes, which might be hidden by apparent similarities in the clonal evolution architecture, could partially account for the observed clinical heterogeneity which cannot be fully explained by genetics..$^{90}$ Developing tools to stratify patients based on natural selection or neutral evolution could help understand and better anticipate these different clinical paths.

In addition to cancer treatment, the role of neutral evolution might also impact the interpretation of and intervention in clonal hematopoiesis. ${ }^{91}$ While clones have been retrospectively identified several years before the diagnosis of $\mathrm{AML}^{92,93}$ suggesting a potential window of therapeutic intervention to avoid transformation of clonal hematopoiesis to $\mathrm{AML}$, most instances of clonal hematopoiesis remain benign ( $\mathrm{AML}$ driver mutations are ubiquitously found in adults $\left.{ }^{94}\right)$. Being able to discriminate clonal hematopoiesis emerging by neutral evolution from that emerging because of natural selection could contribute to a better understanding of the various natural histories and a better identification of patients at risk of malignant transformation. 


\section{Conclusion and perspective}

Whilst it is tempting to assume that the linear evolution observed in blood cancers is caused by natural selection acting on fitter clones, we should not ignore the possibility that linear evolution can result from neutral evolution through genetic drift if the number of contributing SCs is sufficiently low. To discriminate which of natural selection or genetic drift is causing linear architecture in blood cancer, further investigations into the number of SCS contributing to clonal evolution on the long term in different contexts (aging, cancer) are required.

In cancers, in the absence of mechanistic studies demonstrating a gain of fitness, natural selection can only be inferred indirectly, from the observation of large clones and/or from the recurrence of mutations across patients. However, in the case of indirect evidence for positive selection, alternatives causes are also possible. When large clones are observed, they could also result from genetic drift if the population of SCs is small enough. When recurrent mutations are observed across patients, the mutations could be selected for, or alternatively, they could occur more frequently (passenger hotspot). ${ }^{15,16}$

In practice, genetic drift could explain the presence of clones with no candidate driver mutations observed in some hematological malignancies, e.g. $10 \%$ of myeloproliferative neoplasms, ${ }^{95,96}$ although it is always possible that genetic or epigenetic driver alterations have been undetected. ${ }^{63}$ In addition, genetic drift could explain growth of clones with mutations providing no clear fitness advantage to stem cells such as JAK2 ${ }^{\mathrm{V} 617 \mathrm{~F}, 97}$ or SRSF2 $^{\mathrm{P} 95 \mathrm{H}}$ mutations, $^{98}$ but others factors could be involved such as the microenvironment, epimutations or non-coding mutations. To evaluate the plausibility of genetic drift among these possibilities, a better knowledge of the number of contributing SCs and of the personal history of the patient (whether they might have suffered SCs exhaustion) seems crucial.

Importantly, genetic drift can indirectly increase the risk of malignancies. Genetic drift could allow a mutation with no selective advantage in SCs but a malignant phenotype in downstream cells to expand in the SC compartment. When genetic drift is strong enough to favor linear evolution, it increases the likelihood of accumulating mutations, among which potentially malignant combinations of mutations. Both processes might have important implications for cancer risk and management.

To conclude, we wish to encourage the community to question the general assumption that clonal evolution by natural selection is the only possible driving force of blood cancer. It is important to consider other hypotheses so as to avoid the potential misinterpretation of observed phenomena such as large clones. In addition, such assumptions have consequences for patients. For example, evolutionary steering strategies try to predict the course of evolution to avoid therapy escape but may not work well for neutrally evolving tumors. These hypotheses could also be relevant in the interpretation of minimal residual disease, the late reoccurrence of clones, and the phenomenon of clonal hematopoiesis. It is thus important to acknowledge that clonal evolution can occur without natural selection even in instances of linear evolution and/or presence of recurrent mutations, and to be able to distinguish which clones have expanded as a result of genetic drift and which as a result of selection. 


\section{Acknowledgments}

We would like to thank Jason Cosgrove, Olivier Delattre, Matthieu Duchmann, Efe Ilker, Arnaud Pocheville, Celine Vallot, and Cristina Lo Celso for their feedback on our manuscript. We would like to thank Jamie Blundell and the other two anonymous reviewers for their feedbacks on this manuscript. The study was supported by an ATIP-Avenir grant from CNRS and Bettencourt-Schueller Foundation (to L.P.) and two grants from the Labex CelTisPhyBio (ANR10-LBX-0038) and Idex Paris-Science-Lettres Program (ANR-10-IDEX-0001-02 PSL) (to L.P.). It was also supported by a starting ERC grant from the H2020 program (758170-Microbar to L.P.) and a James S. McDonnell Foundation grant (220020480.01 to L.L.).

\section{Authorship}

AML performed the simulation study with input from LP and LL. Based on LL initial idea, all the authors have conceived the study and written the manuscript.

\section{Conflict-of-interest statements}

The authors declare no conflict of interest. 


\section{References}

1. McGranahan N, Swanton C. Clonal Heterogeneity and Tumor Evolution: Past, Present, and the Future. Cell. 2017;168(4):613-628.

2. Andor N, Graham TA, Jansen M, et al. Pan-cancer analysis of the extent and consequences of intratumor heterogeneity. Nat. Med. 2016;22(1):105-113.

3. Bailey MH, Tokheim C, Porta-Pardo E, et al. Comprehensive Characterization of Cancer Driver Genes and Mutations. Cell. 2018;173(2):371-385.e18.

4. Tamborero D, Dienstmann R, Rachid MH, et al. Support systems to guide clinical decision-making in precision oncology: The Cancer Core Europe Molecular Tumor Board Portal. Nat. Med. 2020;26(7):992994.

5. Steensma DP. How I use molecular genetic tests to evaluate patients who have or may have myelodysplastic syndromes. Blood. 2018;132(16):1657-1663.

6. Ling S, Hu Z, Yang Z, et al. Extremely high genetic diversity in a single tumor points to prevalence of nonDarwinian cell evolution. Proc. Natl. Acad. Sci. 2015;112(47):E6496-6505.

7. Sottoriva A, Kang H, Ma Z, et al. A Big Bang model of human colorectal tumor growth. Nat. Genet. 2015;47(3):209-216.

8. Williams MJ, Werner B, Barnes CP, Graham TA, Sottoriva A. Identification of neutral tumor evolution across cancer types. Nat. Genet. 2016;48(3):238-244.

9. Itzykson R, Solary E. An evolutionary perspective on chronic myelomonocytic leukemia. Leukemia. 2013;27(7):1441-50.

10. Itzykson R, Kosmider O, Renneville A, et al. Clonal architecture of chronic myelomonocytic leukemias. Blood. 2013;121(12):2186-2198.

11. Johnson DC, Lenive O, Mitchell J, et al. Neutral tumor evolution in myeloma is associated with poor prognosis. Blood. 2017;130(14):1639-1643.

12. Campbell PJ, Getz G, Korbel JO, et al. Pan-cancer analysis of whole genomes. Nature. 2020;578(7793):8293.

13. Bohannan ZS, Mitrofanova A. Calling Variants in the Clinic: Informed Variant Calling Decisions Based on Biological, Clinical, and Laboratory Variables. Comput. Struct. Biotechnol. J. 2019;17:561-569.

14. Plutynski A. The Cancer Genome Atlas Project: Data-driven, Hypothesis-driven or Something In-Between? Perspect. Hum. Genome Proj. Genomics. 2021;

15. Buisson R, Langenbucher A, Bowen D, et al. Passenger hotspot mutations in cancer driven by APOBEC3A and mesoscale genomic features. Science (80-. ). 2019;364(6447):eaaw2872.

16. Hess JM, Bernards A, Kim J, et al. Passenger Hotspot Mutations in Cancer. Cancer Cell. 2019;36(3):288301.e14.

17. Sun R, Hu Z, Curtis C. Big bang tumor growth and clonal evolution. Cold Spring Harb. Perspect. Med. 2018;8(5):a028381.

18. Cross WC, Graham TA, Wright NA. New paradigms in clonal evolution: punctuated equilibrium in cancer. J. Pathol. 2016;240(2):126-136.

19. Davis A, Gao R, Navin N. Tumor evolution: Linear, branching, neutral or punctuated? Biochim. Biophys. Acta-Rev. Cancer. 2017;1867(2):151-161.

20. Chesnais V, Arcangeli M-L, Delette C, et al. Architectural and functional heterogeneity of hematopoietic stem/progenitor cells in non-del(5q) myelodysplastic syndromes. Blood. 2017;129(4):484-496.

21. Merlevede J, Droin N, Qin T, et al. Mutation allele burden remains unchanged in chronic myelomonocytic leukaemia responding to hypomethylating agents. Nat. Commun. 2016;7:10767.

22. Hirsch P, Zhang Y, Tang R, et al. Genetic hierarchy and temporal variegation in the clonal history of acute myeloid leukaemia. Nat. Commun. 2016;7:12475.

23. Potter N, Miraki-Moud F, Ermini L, et al. Single cell analysis of clonal architecture in acute myeloid leukaemia. Leukemia. 2019;33(5):1113-1123.

24. da Silva-Coelho P, Kroeze LI, Yoshida K, et al. Clonal evolution in myelodysplastic syndromes. Nat. Commun. 2017;8:15099.

25. Makishima H, Yoshizato T, Yoshida K, et al. Dynamics of clonal evolution in myelodysplastic syndromes. Nat. Genet. 2017;49(2):204-212.

26. Lundberg P, Karow A, Nienhold R, et al. Clonal evolution and clinical correlates of somatic mutations in myeloproliferative neoplasms. Blood. 2014;123(14):2220-8.

27. Melchor L, Brioli A, Wardell CP, et al. Single-cell genetic analysis reveals the composition of initiating clones and phylogenetic patterns of branching and parallel evolution in myeloma. Leukemia.

2014;28(8):1705-15. 
28. Caye A, Strullu M, Guidez F, et al. Juvenile myelomonocytic leukemia displays mutations in components of the RAS pathway and the PRC2 network. Nat. Genet. 2015;47(11):1334-1340.

29. Landau DA, Carter SL, Stojanov P, et al. Evolution and impact of subclonal mutations in chronic lymphocytic leukemia. Cell. 2013;152(4):714-26.

30. Cerrano M, Duchmann M, Kim R, et al. Clonal dominance is an adverse prognostic factor in acute myeloid leukemia treated with intensive chemotherapy. Leukemia. 2020;1-12.

31. Itzykson R, Duployez N, Fasan A, et al. Clonal interference of signaling mutations worsens prognosis in core-binding factor acute myeloid leukemia. Blood. 2018;132(2):187-196.

32. Ding L, Ley TJ, Larson DE, et al. Clonal evolution in relapsed acute myeloid leukaemia revealed by wholegenome sequencing. Nature. 2012;481(7382):506-10.

33. Jacoby MA, Duncavage EJ, Chang GS, et al. Subclones dominate at MDS progression following allogeneic hematopoietic cell transplant. JCl insight. 2018;3(5):e98962.

34. Theocharides A, Boissinot M, Girodon F, et al. Leukemic blasts in transformed JAK2-V617F-positive myeloproliferative disorders are frequently negative for the JAK2-V617F mutation. Blood.

2007;110(1):375-379.

35. Venkatesan S, Swanton C. Tumor Evolutionary Principles: How Intratumor Heterogeneity Influences Cancer Treatment and Outcome. Am. Soc. Clin. Oncol. Educ. B. 2016;36:e141-e149.

36. Sottoriva A, Barnes CP, Graham TA. Catch my drift? Making sense of genomic intra-tumour heterogeneity. Biochim. Biophys. Acta - Rev. Cancer. 2017;1867(2):95-100.

37. Lynch M, Ackerman MS, Gout J-F, et al. Genetic drift, selection and the evolution of the mutation rate. Nat. Rev. Genet. 2016;17(11):704-714.

38. Sottoriva A, Verhoeff JJ, Borovski T, et al. Cancer stem cell tumor model reveals invasive morphology and increased phenotypical heterogeneity. Cancer Res. 2010;70(1):46-56.

39. Greaves M. Cancer stem cells as "units of selection." Evol Appl. 2013;6(1):102-108.

40. Lohr JG, Stojanov P, Carter SL, et al. Widespread genetic heterogeneity in multiple myeloma: implications for targeted therapy. Cancer Cell. 2014;25(1):91-101.

41. Bozic I, Paterson C, Waclaw B. On measuring selection in cancer from subclonal mutation frequencies. PLOS Comput. Biol. 2019;15(9):e1007368.

42. Lee-Six H, Øbro NF, Shepherd MS, et al. Population dynamics of normal human blood inferred from somatic mutations. Nature. 2018;561(7724):473-478.

43. Watson CJ, Papula AL, Poon GYP, et al. The evolutionary dynamics and fitness landscape of clonal hematopoiesis. Science (80-. ). 2020;367(6485):1449-1454.

44. Dingli D, Pacheco JM. Allometric Scaling of the Active Hematopoietic Stem Cell Pool across Mammals. PLoS One. 2006;1(1):e2.

45. Wu S, Powers S, Zhu W, Hannun YA. Substantial contribution of extrinsic risk factors to cancer development. Nature. 2015;

46. Abkowitz JL, Catlin SN, McCallie MT, Guttorp P. Evidence that the number of hematopoietic stem cells per animal is conserved in mammals. Blood. 2002;100(7):2665-2667.

47. Holstege H, Pfeiffer W, Sie D, et al. Somatic mutations found in the healthy blood compartment of a 115yr-old woman demonstrate oligoclonal hematopoiesis. Genome Res. 2014;24(5):733-42.

48. de Haan G, Lazare S. Aging of hematopoietic stem cells. Blood. 2017;131(5):479-487.

49. Goodell MA, Rando TA. Stem cells and healthy aging. Science (80-. ). 2015;350(6265):1199-1204.

50. Ganuza M, Hall T, Finkelstein D, et al. The global clonal complexity of the murine blood system declines throughout life and after serial transplantation. Blood. 2019;133(18):1927-1942.

51. Säwen P, Eldeeb M, Erlandsson E, et al. Murine HSCs contribute actively to native hematopoiesis but with reduced differentiation capacity upon aging. Elife. 2018;7:e41258.

52. Hu T, Kitano A, Luu V, et al. Bmi1 Suppresses Adipogenesis in the Hematopoietic Stem Cell Niche. Stem Cell Reports. 2019;13(3):545-558.

53. Naveiras $\mathrm{O}$, Nardi $\mathrm{V}$, Wenzel PL, et al. Bone-marrow adipocytes as negative regulators of the haematopoietic microenvironment. Nature. 2009;460(7252):259-263.

54. Hirche C, Frenz T, Haas SF, et al. Systemic Virus Infections Differentially Modulate Cell Cycle State and Functionality of Long-Term Hematopoietic Stem Cells In Vivo. Cell Rep. 2017;19(11):2345-2356.

55. Matatall KA, Jeong M, Chen S, et al. Chronic Infection Depletes Hematopoietic Stem Cells through StressInduced Terminal Differentiation. Cell Rep. 2016;17(10):2584-2595.

56. Medinger M, Drexler B, Lengerke C, Passweg J. Pathogenesis of Acquired Aplastic Anemia and the Role of the Bone Marrow Microenvironment. Front. Oncol. 2018;8:587.

57. Shao L, Feng W, Li H, et al. Total body irradiation causes long-term mouse BM injury via induction of HSC 
premature senescence in an Ink4a- and Arf-independent manner. Blood. 2014;123(20):3105-3115.

58. King KY, Huang Y, Nakada D, Goodell MA. Environmental influences on clonal hematopoiesis. Exp. Hematol. 2020;83:66-73.

59. Cook EK, Luo M, Rauh MJ. Clonal hematopoiesis and inflammation: Partners in leukemogenesis and comorbidity. Exp. Hematol. 2020;83(0):85-94.

60. Ganuza M, Hall T, Obeng EA, McKinney-Freeman S. Clones assemble! The clonal complexity of blood during ontogeny and disease. Exp. Hematol. 2020;83(0):35-47.

61. Genovese G, Kahler AK, Handsaker RE, et al. Clonal hematopoiesis and blood-cancer risk inferred from blood DNA sequence. N Engl J Med. 2014;371(26):2477-2487.

62. Zink F, Stacey SN, Norddahl GL, et al. Clonal hematopoiesis, with and without candidate driver mutations, is common in the elderly. Blood. 2017;130(6):742-752.

63. Poon G, Watson CJ, Fisher DS, Blundell JR. Synonymous mutations reveal genome-wide driver mutation rates in healthy tissues. bioRxiv. 2020;2020.10.08.331405.

64. Aiuti A, Biasco L, Scaramuzza S, et al. Lentiviral hematopoietic stem cell gene therapy in patients with Wiskott-Aldrich syndrome. Science. 2013;341(6148):1233151.

65. Biasco L, Pellin D, Scala S, et al. In Vivo Tracking of Human Hematopoiesis Reveals Patterns of Clonal Dynamics during Early and Steady-State Reconstitution Phases. 2016.

66. Wong WH, Bhatt S, Trinkaus K, et al. Engraftment of rare, pathogenic donor hematopoietic mutations in unrelated hematopoietic stem cell transplantation. Sci. Transl. Med. 2020;12(526):

67. Frick $\mathrm{M}$, Chan $\mathrm{W}$, Arends $\mathrm{CM}$, et al. Role of donor clonal hematopoiesis in allogeneic hematopoietic stemcell transplantation. J. Clin. Oncol. 2019;37(5):375-385.

68. Boettcher S, Wilk CM, Singer J, et al. Clonal hematopoiesis in donors and long-term survivors of related allogeneic hematopoietic stem cell transplantation. Blood. 2020;135(18):1548-1559.

69. Gibson CJ, Kennedy JA, Nikiforow S, et al. Donor-engrafted CHIP is common among stem cell transplant recipients with unexplained cytopenias. Blood. 2017;130(1):91-94.

70. Wilson A, Laurenti E, Oser G, et al. Hematopoietic stem cells reversibly switch from dormancy to selfrenewal during homeostasis and repair. Cell. 2008;135(6):1118-1129.

71. Notta F, Doulatov S, Laurenti E, et al. Isolation of single human hematopoietic stem cells capable of longterm multilineage engraftment. Science (80-. ). 2011;333(6039):218-221.

72. Benz C, Copley MR, Kent DG, et al. Hematopoietic Stem Cell Subtypes Expand Differentially during Development and Display Distinct Lymphopoietic Programs. Cell Stem Cell. 2012;10(3):273-283.

73. Oguro H, Ding L, Morrison SJ. SLAM Family Markers Resolve Functionally Distinct Subpopulations of Hematopoietic Stem Cells and Multipotent Progenitors. Cell Stem Cell. 2013;13(1):102-116.

74. Sanjuan-Pla A, Macaulay IC, Jensen CT, et al. Platelet-biased stem cells reside at the apex of the haematopoietic stem-cell hierarchy. Nature. 2013;502(7470):232-236.

75. Dykstra B, Kent D, Bowie M, et al. Long-Term Propagation of Distinct Hematopoietic Differentiation Programs In Vivo. Cell Stem Cell. 2007;1(2):218-229.

76. Karamitros D, Stoilova B, Aboukhalil Z, et al. Single-cell analysis reveals the continuum of human lymphomyeloid progenitor cells. Nat. Immunol. 2018;19(1):85-97.

77. Laurenti E, Frelin C, Xie S, et al. CDK6 Levels Regulate Quiescence Exit in Human Hematopoietic Stem Cells. Cell Stem Cell. 2015;16(3):302-313.

78. Bernitz JM, Kim HS, MacArthur B, Sieburg H, Moore K. Hematopoietic Stem Cells Count and Remember Self-Renewal Divisions. Cell. 2016;167(5):1296-1309.e10.

79. Morcos MNF, Zerjatke T, Glauche I, et al. Continuous mitotic activity of primitive hematopoietic stem cells in adult mice. J. Exp. Med. 2020;217(6):e20191284.

80. Sun J, Ramos A, Chapman B, et al. Clonal dynamics of native haematopoiesis. Nature. 2014;514(7522):322-327.

81. Busch K, Klapproth K, Barile M, et al. Fundamental properties of unperturbed haematopoiesis from stem cells in vivo. Nature. 2015;518(7540):542-546.

82. Sawai CM, Babovic S, Upadhaya S, et al. Hematopoietic Stem Cells Are the Major Source of Multilineage Hematopoiesis in Adult Animals. Immunity. 2016;45(3):597-609.

83. Schoedel KB, Morcos MNF, Zerjatke T, et al. The bulk of the hematopoietic stem cell population is dispensable for murine steady-state and stress hematopoiesis. Blood. 2016;128(19):2285-2297.

84. Sheikh BN, Yang Y, Schreuder J, et al. MOZ (KAT6A) is essential for the maintenance of classically defined adult hematopoietic stem cells. Blood. 2016;128(19):2307-2318.

85. Duarte D, Amarteifio S, Ang H, et al. Defining the in vivo characteristics of acute myeloid leukemia cells behavior by intravital imaging. Immunol. Cell Biol. 2019;97(2):229-235. 
86. Luskin MR, Murakami MA, Manalis SR, Weinstock DM. Targeting minimal residual disease: a path to cure? Nat. Rev. Cancer. 2018;18(4):255-263.

87. Gatenby RA, Silva AS, Gillies RJ, Frieden BR. Adaptive therapy. Cancer Res. 2009;69(11):4894-903.

88. Zhang J, Cunningham JJ, Brown JS, Gatenby RA. Integrating evolutionary dynamics into treatment of metastatic castrate-resistant prostate cancer. Nat. Commun. 2017;8(1):1816.

89. Acar A, Nichol D, Fernandez-Mateos J, et al. Exploiting evolutionary steering to induce collateral drug sensitivity in cancer. Nat. Commun. 2020;11(1):1923.

90. Ball M, List AF, Padron E. When clinical heterogeneity exceeds genetic heterogeneity: Thinking outside the genomic box in chronic myelomonocytic leukemia. Blood. 2016;128(20):2381-2387.

91. Steensma DP. Clinical Implications of Clonal Hematopoiesis. Mayo Clin. Proc. 2018;93(8):1122-1130.

92. Desai P, Mencia-Trinchant N, Savenkov O, et al. Somatic mutations precede acute myeloid leukemia years before diagnosis. Nat. Med. 2018;24(7):1015-1023.

93. Abelson S, Collord G, Ng SWK, et al. Prediction of acute myeloid leukaemia risk in healthy individuals. Nature. 2018;559(7714):400-404.

94. Young AL, Challen GA, Birmann BM, Druley TE. Clonal haematopoiesis harbouring AML-associated mutations is ubiquitous in healthy adults. Nat. Commun. 2016;7:12484.

95. Grinfeld J, Nangalia J, Baxter EJ, et al. Classification and Personalized Prognosis in Myeloproliferative Neoplasms. N. Engl. J. Med. 2018;379(15):1416-1430.

96. Williams N, Lee J, Moore $\mathrm{L}$, et al. Phylogenetic reconstruction of myeloproliferative neoplasm reveals very early origins and lifelong evolution. bioRxiv. 2020;2020.11.09.374710.

97. Kent DG, Li J, Tanna H, et al. Self-Renewal of Single Mouse Hematopoietic Stem Cells Is Reduced by JAK2V617F Without Compromising Progenitor Cell Expansion. PLoS Biol. 2013;13(3):e1002092.

98. Zheng X, Carstens JL, Kim J, et al. Epithelial-to-mesenchymal transition is dispensable for metastasis but induces chemoresistance in pancreatic cancer. Nature. 2015;527(7579):525-530.

99. R Core Team. R: A language and environment for statistical computing. Vienna, Austria: R Foundation for Statistical Computing; 2014.

100. Miller CA, McMichael J, Dang HX, et al. Visualizing tumor evolution with the fishplot package for R. BMC Genomics. 2016;17(1):880. 

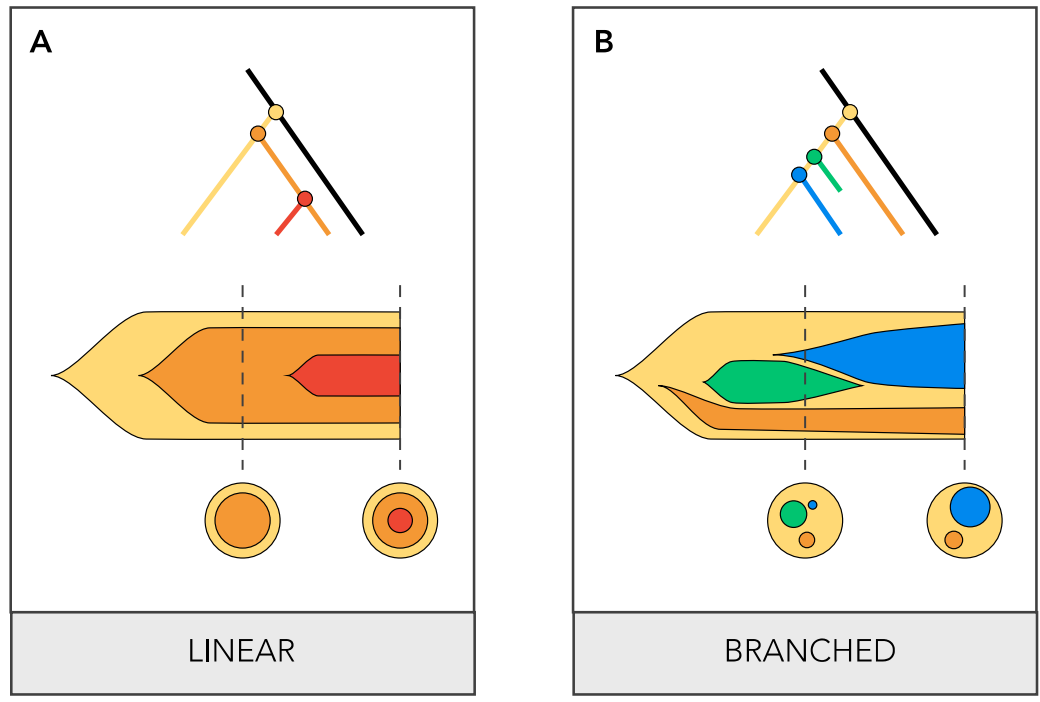

Figure 1. Clonal architecture can follow two types of organization: linear and branched evolution. (A) Linear evolution refers to a Russian doll-like organization where each new clone occurs inside the previous one. Each new clone thus inherits all the previous mutations. (B) Branched evolution refers to parallel evolution of different clones. These two clonal architectures can be represented in various ways including phylogenetic trees that reconstruct the occurrence of mutations through time (top), fishplots that, in addition, represent the growth of each new clone through time (middle), or slices that capture the clonal architecture similarly to the fishplot but at a particular time point (bottom). 
A

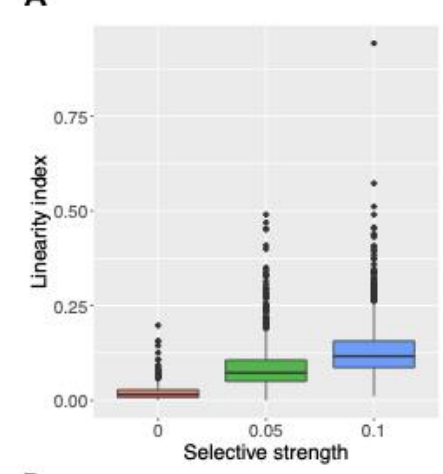

D

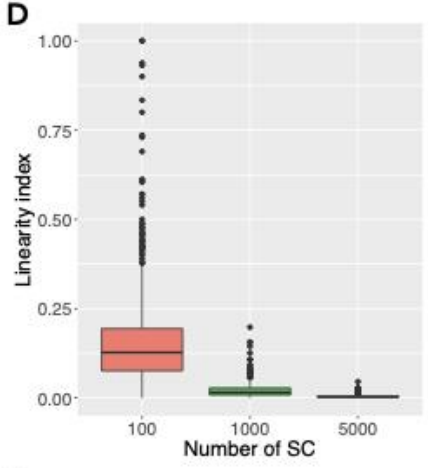

$\mathbf{F}$

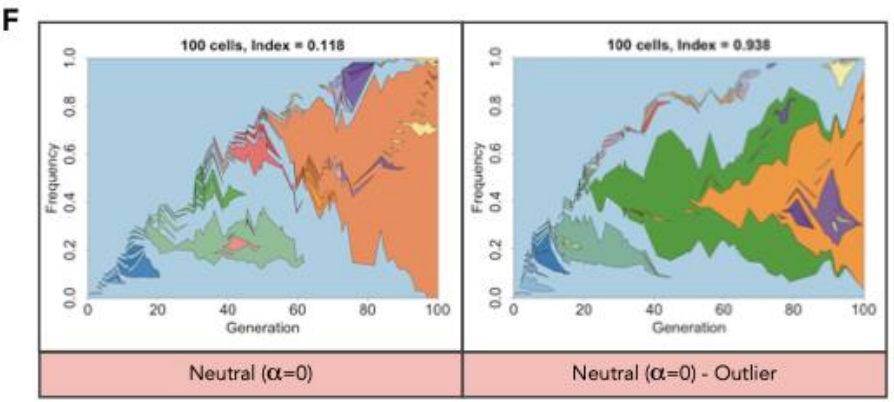

C
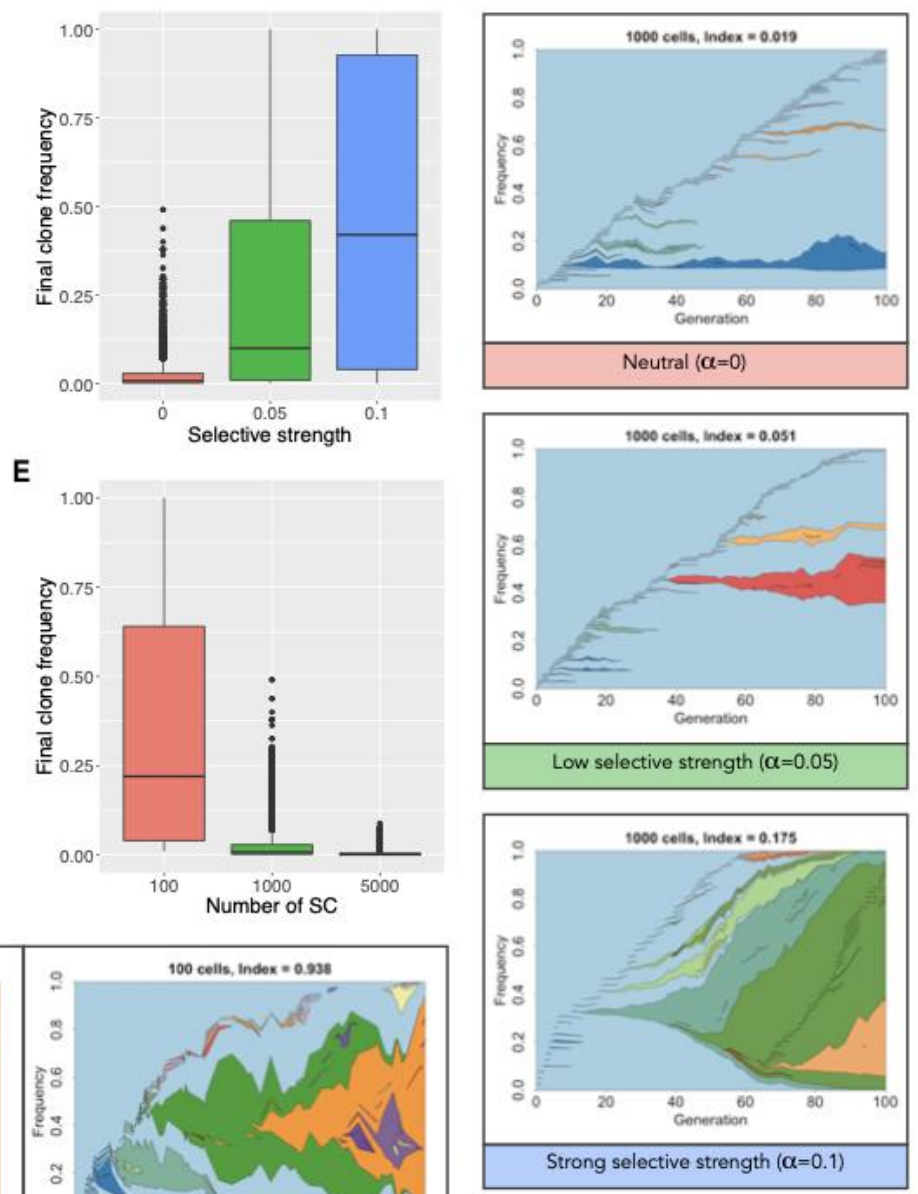

Figure 2. Mathematical modeling of clonal evolution illustrates that linear evolution requires natural selection to occur in large populations but can occur through drift in small populations. (A-C) Simulations of a Moran model with 1000 cells, and with increasing selective strength (increasing probability to self-renew upon acquisition of a mutation, $\alpha$ ). When $\alpha=0$, evolution is neutral, all cells have equal probability to self-renew whether mutant or not. When $\alpha>0$ then mutated cells have an increased probability to expand, i.e. mutations increase their fitness. (A) Linearity indices at $t=100$ for selective strengths $\alpha=0, \alpha=0.05$ and $\alpha=0.1$. (B) Final clone frequency for all remaining clones across all simulations at $t=100$ for selective strengths $\alpha=0, \alpha=0.05$ and $\alpha=0.1$. (C) Representative examples of clonal evolution in these simulations. The text above indicates the numbers of cells remaining and the linearity index at the end of the simulation. (D-F) Simulations with neutral evolution $(\alpha=0)$ for 100, 1000, and 5000 SCS. (D) Linearity indices at $\mathrm{t}=100$ for 100, 1000, and 5000 SCs. (E) Final clone frequency for all remaining clones across all simulations at $\mathrm{t}=100$ for 100, 1000, and 5000 SCs. (F) Representative example of clonal evolution for 100 cells, and one example of an outlier with a high linearity index. 
A
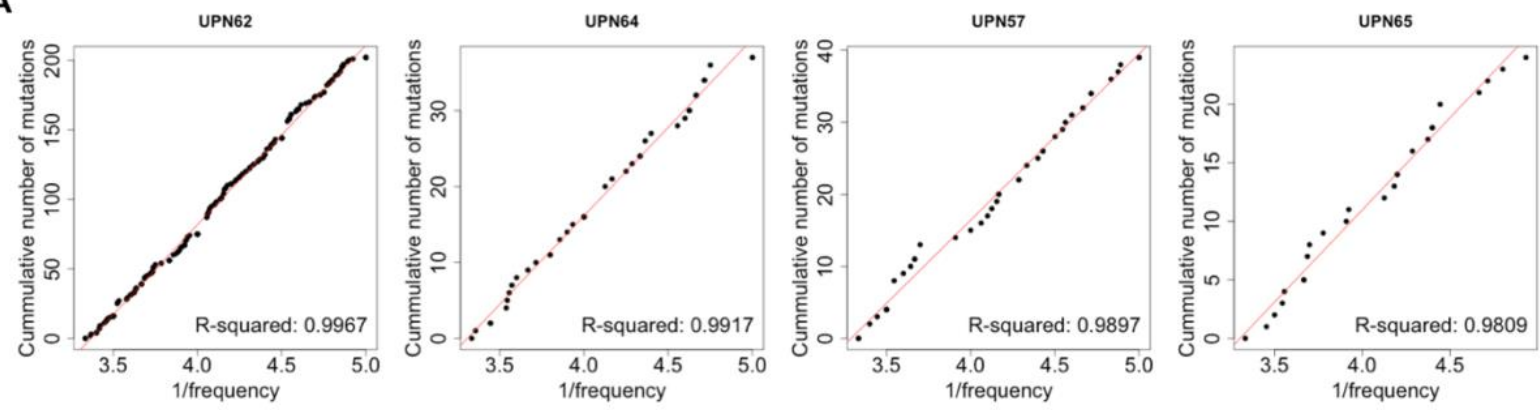

B

\begin{tabular}{|c|c|c|c|c|c|c|c|}
\hline $\begin{array}{l}\text { tumor_} \\
\text { reads_Ref }\end{array}$ & $\begin{array}{l}\text { tumor___ } \\
\text { reads_Var }\end{array}$ & $\begin{array}{l}\text { tumor__ } \\
\text { var_Frq }\end{array}$ & $\begin{array}{l}\text { somatic_ } \\
\text { pvalue }\end{array}$ & Func & Gene & ExonicFunc & Predictions \\
\hline 32 & 9 & $21,95 \%$ & 0.014803 & $\begin{array}{l}\text { exonic; } \\
\text { splicing }\end{array}$ & POLR3A & $\begin{array}{l}\text { nonsynony- } \\
\text { mous SNV }\end{array}$ & D; D; D; D \\
\hline 48 & 12 & $20 \%$ & 0.001722 & exonic & KIF20B & $\begin{array}{l}\text { synonymous } \\
\text { SNV }\end{array}$ & \\
\hline 34 & 12 & $26,09 \%$ & 0.000759 & exonic & ITSN1 & $\begin{array}{l}\text { nonsynony- } \\
\text { mous SNV }\end{array}$ & D; NA; D; N \\
\hline 29 & 23 & $44,23 \%$ & $1.0191 \mathrm{e}-06$ & exonic & TET2 & $\begin{array}{l}\text { frameshift } \\
\text { deletion }\end{array}$ & $\mathrm{NA} ; \mathrm{NA} ; \mathrm{NA} ; \mathrm{NA}$ \\
\hline 27 & 11 & $28,95 \%$ & 0.001618 & $\begin{array}{l}\text { exonic; } \\
\text { splicing }\end{array}$ & CUL9 & $\begin{array}{l}\text { nonsynony- } \\
\text { mous SNV }\end{array}$ & D; D; D; D \\
\hline 31 & 12 & $27,91 \%$ & 0.000494 & $\begin{array}{l}\text { exonic; } \\
\text { splicing }\end{array}$ & PRRC2B & $\begin{array}{l}\text { nonsynony- } \\
\text { mous SNV }\end{array}$ & \\
\hline
\end{tabular}

C

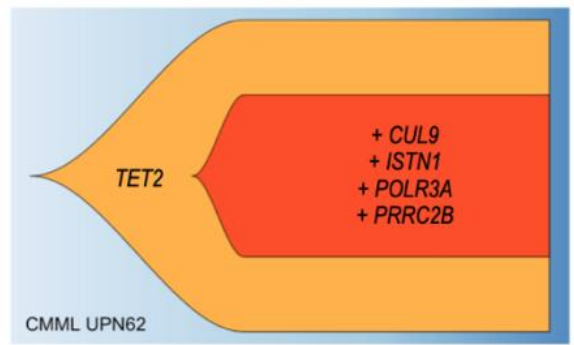

Figure 3. Neutral evolution in a CMML patient with linear evolution. (A). Inverse mutation allele frequency versus cumulative number of mutations from CMML patient tumors from ${ }^{21}$. The R-squared value was computed as in ${ }^{11}$, filtering mutations outside the frequency range of $[0.12,0.3]$. R-squared greater than 0.98 were taken as samples compatible with neutral evolution and displayed. (B) Table listing exonic mutations of patient UPN62. This information has been extracted from ${ }^{21}$ which reports results from whole genome sequencing of $17 \mathrm{CMML}$ patients. The final column states the impact of the mutation as predicted by ${ }^{21}$. $\mathrm{D}$ indicates that the mutation is predicted to be damaging and $\mathrm{N}$ that the mutation is predicted to be neutral. (C) Fishplot ${ }^{99,100}$ representing the clonal architecture of the patient with the putative driver mutations (TET2 mutations are present around $60 \%$ of CMML patients, and mutations of PRRC2B have been recently identified as recurrent in CMML patients ${ }^{21}$ ). Drawn in R using the fishplot library. ${ }^{99,100}$ 